\title{
A TRANSONIC POTENTIAL SOLVER WITH AN EMBEDDED WAKE APPROACH USING MULTIVALUED FINITE ELEMENTS
}

\author{
Iñigo López ${ }^{* \dagger}$, Eloisa Baez Jones ${ }^{\dagger}$, Marc Núñez $^{\ddagger}$, Rubén Zorrilla $^{\ddagger}$, \\ Riccardo Rossi $^{\ddagger}$, Kai-Uwe Bletzinger ${ }^{\dagger}$ and Roland Wüchner ${ }^{\dagger}$ \\ $\dagger$ Chair of Structural Analysis \\ Technical University of Munich \\ Arcisstr. 21, 80333 Munich, Germany \\ e-mail: inigo.lopez@tum.de, kub@tum.de,wuechner@tum.de \\ $\ddagger$ Centre Internacional de Metodes Numerics en Enginyeria (CIMNE) \\ Universidad Politécnica de Cataluña \\ Campus Norte UPC, 08034 Barcelona, Spain \\ e-mail: mnunez@cimne.upc.edu,rzorrilla@cimne.upc.edu, rrossi@cimne.upc.edu
}

Key words: Embedded Wake Surfaces, Full-Potential Equation, Transonic Flow, FiniteElement Method, Aeroelastic Optimization

\begin{abstract}
A potential transonic solver with an embedded wake is presented. The flow outside of attached boundary layers of streamlined bodies flying at high Reynold numbers can be assumed to be irrotational and isentropic. This assumption reduces the NavierStokes equations to a single scalar nonlinear partial differential equation, namely the full-potential equation (FPE). The FPE expresses the conservation of mass in terms of the velocity potential. In this work, the FPE is discretized using a standard Galerkin finite element method, and the nonlinear system of equations stemming from the discretization is solved using Newton's method. An artificial compressibility method is used to stabilize the problem in supersonic flow regions. This method prevents the Jacobian from becoming singular and allows capturing shock waves. To include the viscosity effects in the lift generation, a model for the trailing wake needs to be introduced. In the presented method, the wake is modeled as a straight surface in the free-stream direction. This assumption is relaxed allowing mass flux across the wake. To capture the discontinuity in the velocity potential across the wake, a multivalued element method is employed. This implicit description of the wake within the mesh presents an effective approach to perform fluidstructure interaction computations and apply aeroelastic optimization methods, where the position of the wake changes during consecutive iterations. The solver is implemented in Kratos Multi-Physics and verified for different angles of attack and free-stream conditions. Since the pressure does not change in the transverse direction of the boundary layer, the FPE yields accurate lift, induced drag, and moment computations.
\end{abstract}




\section{INTRODUCTION}

Linear potential solvers are still used in the industry to perform aeroelastic optimization in the early design stages. Yet, using higher fidelity solvers could allow detecting design flaws sooner, saving time and money. Full-potential solvers offer an appealing tradeoff, enlarging the range of application to capture nonlinear compressibility effects while providing fast solutions. However, standard full-potential solvers require modeling a gap in the mesh, hindering their effective use for aeroelastic optimization, where the wake's position may change due to the structural response and the geometry's evolutionary steps $[1,2]$. To overcome this problem, an embedded wake approach for potential transonic solvers is proposed.

\section{METHOD}

\section{$2.1 \quad$ Full-potential equation}

\section{The full-potential equation expresses conservation of mass in terms of density $\rho$ and} velocity $u$ :

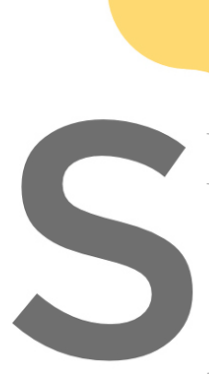

$$
\nabla \cdot(\rho u)=0
$$

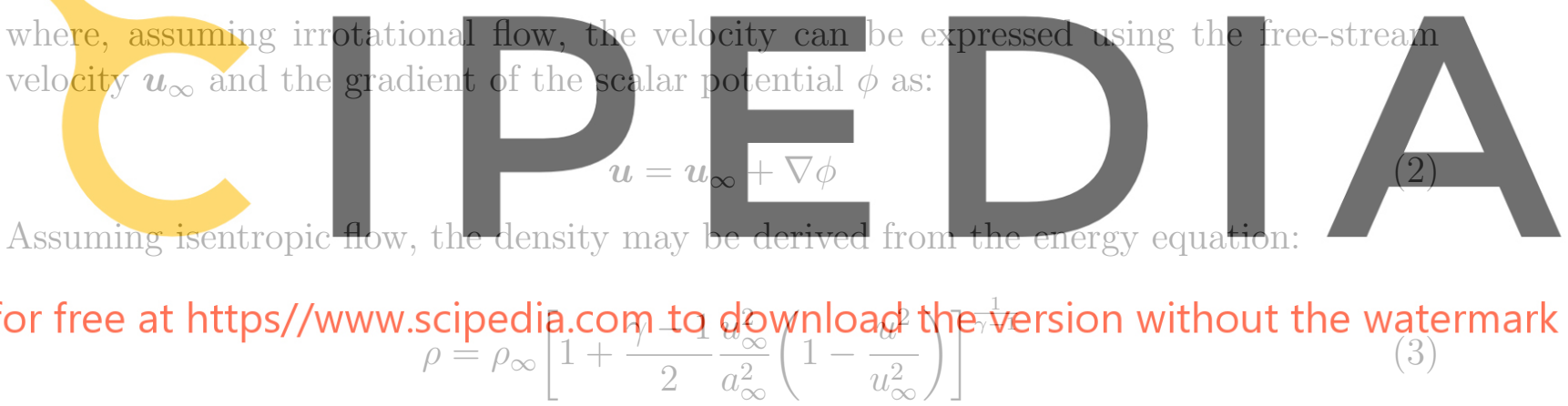

where $\rho_{\infty}$ and $a_{\infty}$ are the free-stream density and speed of sound respectively [3]. The constant $\gamma$ is the heat capacity ratio, which is 1.4 for air.

\subsection{Boundary conditions}

Figure 1 illustrates the fluid domain and its boundaries in two dimensions. The boundary conditions are:

$$
\begin{aligned}
\phi & =\phi_{\infty} & & \text { on } \Gamma_{D} \\
\mathbf{n} \cdot(\rho \boldsymbol{u}) & =g & & \text { on } \Gamma_{N} \\
\mathbf{n} \cdot\left(\rho_{u} \boldsymbol{u}_{u}-\rho_{l} \boldsymbol{u}_{l}\right) & =0 & & \text { on } \Gamma_{W} \\
\left|\boldsymbol{u}_{u}\right|^{2}-\left|\boldsymbol{u}_{l}\right|^{2} & =0 & & \text { on } \Gamma_{W}
\end{aligned}
$$




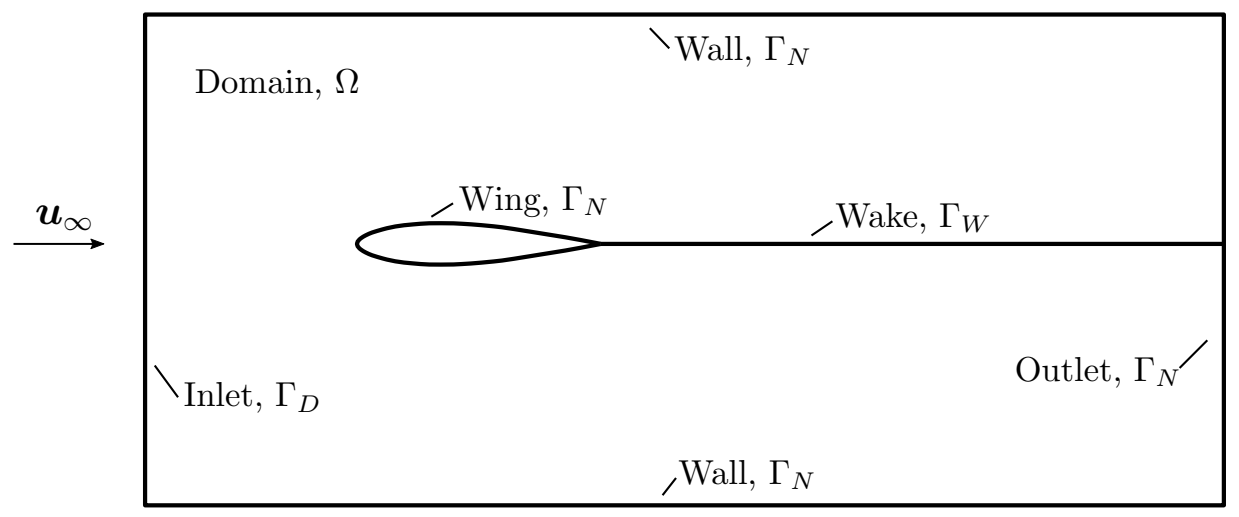

Figure 1: Two-dimensional representation of the fluid domain and its boundaries.

Eq. (4) is a Dirichlet condition, where $\phi_{\infty}$ is an arbitrary value specified along the domain's $\Gamma_{D}$ boundary. This condition is necessary because the full-potential equation and the remaining boundary conditions are only defined in terms of the potential's derivatives.

Because the wake intersects the outlet, and there is a jump in the potential across the wake, a Dirichlet condition cannot be applied at the outlet. Instead, the free-stream

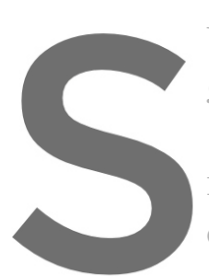
velocity is specified $g=n \cdot\left(\rho u_{\infty}\right)$. A slip

Eqs. (6) and (7) are

for the upper and lowe

direction. To relax thi
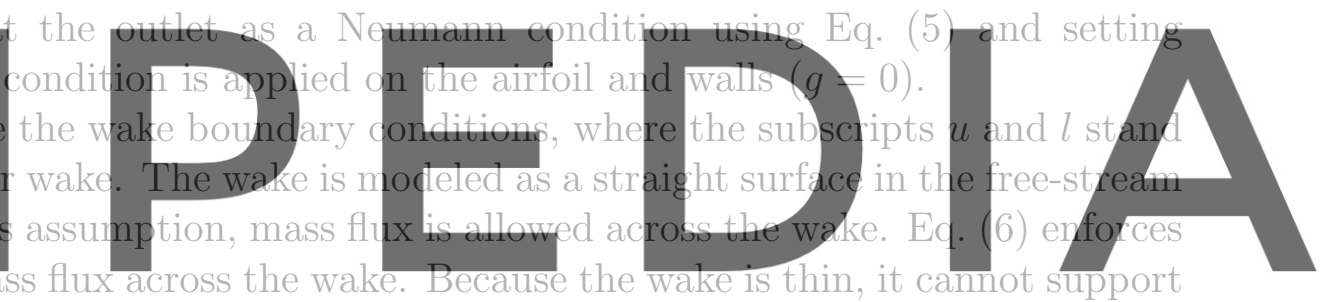

Register for free at https//wWw.scipedia.com to download the version without the watermark

\subsection{Finite element discretization}

Eq. (1) is discretized using a standard Galerkin approach. Here unstructured meshes with linear triangular and tetrahedral elements are used. Weighting Eq. (1) with the test function $N$ and discretizing into finite elements results in:

$$
\sum_{e} \int_{\Omega_{e}} N \nabla \cdot(\rho \boldsymbol{u}) d \Omega_{e}=0
$$

being $\Omega_{e}$ each element's domain. Using integration by parts, applying the divergence theorem, and inserting Eq. (5) yields:

$$
\sum_{e} \int_{\Omega_{e}} \nabla N \cdot(\rho \boldsymbol{u}) d \Omega_{e}=\sum_{c} \int_{\Gamma_{c}} N g d \Gamma_{c}
$$

where $\Gamma_{c}$ is the boundary of element $c$. Eq. (9) can be rewritten in residual form using Einstein notation as: 


$$
R^{i}=\sum_{e} \int_{\Omega_{e}} \frac{\partial N^{i}}{\partial x^{a}} \rho u^{a} d \Omega_{e}-\sum_{c} \int_{\Gamma_{c}} N^{i} g d \Gamma_{c}=0
$$

where $R^{i}$ is the residual at each node. Eq. (10) is solved using Newton's method:

$$
J^{i j} \Delta \phi^{j}=-R^{i}
$$

being $\Delta \phi^{j}$ the solution update and $J^{i j}$ the corresponding Jacobian, which is defined as:

$$
J^{i j}=\frac{\partial R^{i}}{\partial \phi^{j}}=\sum_{e} \int_{\Omega_{e}} \frac{\partial N^{i}}{\partial x^{a}}\left(\rho \frac{\partial u^{a}}{\partial \phi^{j}}+\frac{\partial \rho}{\partial \phi^{j}} u^{a}\right) d \Omega_{e}
$$

\subsection{Artificial compressibility}

To stabilize the problem in supersonic flow regions an artificial compressibility method is employed [4]. This prevents the Jacobian from becoming singular and allows capturing shock waves. In this method, an upwind scheme is used to compute the density:
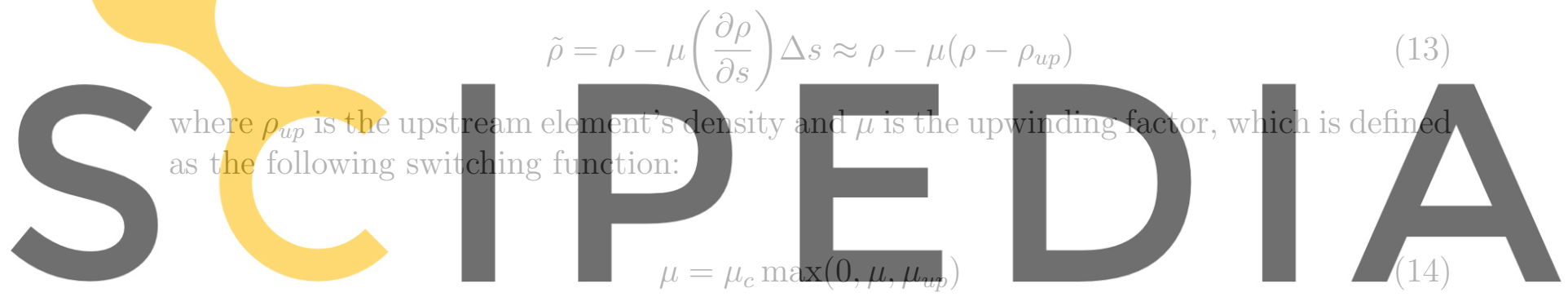

$M_{\text {crit }}^{2}$

Register for free at https//www.scipedial.com to download the version without the watermark

where $\mu_{c}$ is the upwinding factor constant, and $M_{\text {crit }}$ is the critical Mach number. These

factors control the amount of artificial dissipation introduced into the system. Eq. (13) replaces $\rho$ in Eqs. (10) and (12). To avoid reaching vacuum conditions, the fluid variables and their derivatives are clamped to a maximum Mach number squared of $M^{2}=3.0$.

\subsection{Embedded wake approach}

To support a jump in the potential, elements cut by the wake are enriched with auxiliary degrees of freedom and disconnected across the wake, so that wake elements are present twice. This is illustrated in Figure 2, where the auxiliary degrees of freedom are denoted by $\phi_{\text {aux }}^{i}$. The degrees of freedom $\phi_{u}^{i}$ and $\phi_{l}^{i}$ corresponding respectively to the upper and lower wake elements are:

$\phi_{u}^{i}=\left\{\begin{array}{ll}\phi^{i} & \text { if } i^{t h} \text { node above the wake } \\ \phi_{\text {aux }}^{i} & \text { if } i^{t h} \text { node below the wake }\end{array} \phi_{l}^{i}= \begin{cases}\phi_{\text {aux }}^{i} & \text { if } i^{t h} \text { node above the wake } \\ \phi^{i} & \text { if } i^{t h} \text { node below the wake }\end{cases}\right.$ 


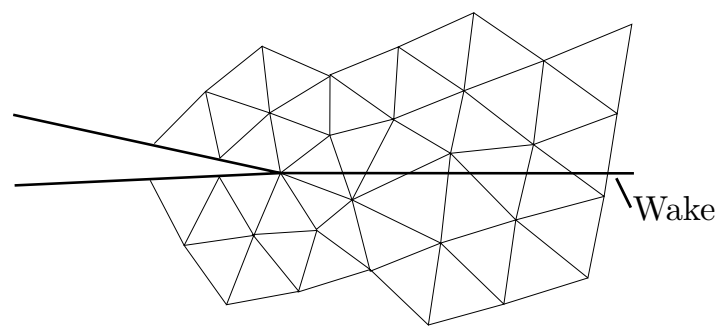

(a) Mesh around the trailing edge.

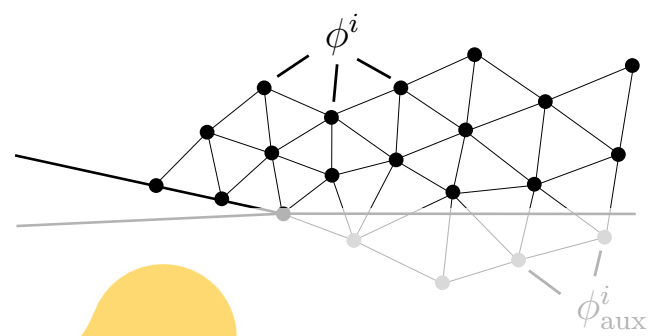

(b) Elements above the wake.

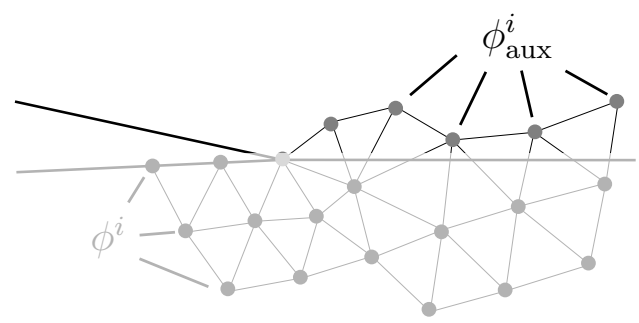

(c) Elements below the wake.

Figure 2: Elements are disconnected across the wake to support a jump in $\phi$.
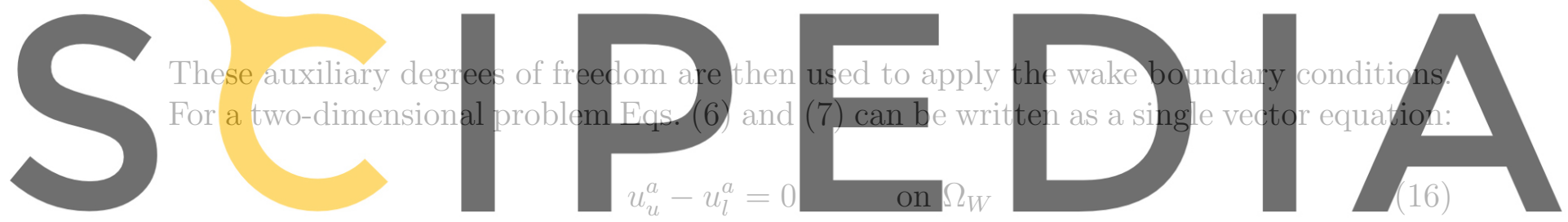

where $\Omega_{W}$ is the domain covered by the wake elements. Applying a least squares finite

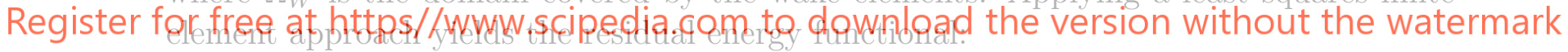

$$
\Pi=\frac{1}{2} \int_{\Omega_{W}}\left(u_{u}^{a}-u_{l}^{a}\right)^{2} d \Omega_{W}
$$

Deriving with respect to the degrees of freedom results in the upper and lower wake conditions' residuals:

$$
\begin{aligned}
& R_{u}^{i}=\frac{\partial \Pi}{\partial \phi_{u}^{i}}=\sum_{W_{u}} \int_{\Omega_{W_{u}}} \frac{\partial N_{u}^{i}}{\partial x^{a}}\left(u_{u}^{a}-u_{l}^{a}\right) d \Omega_{W_{u}} \\
& R_{l}^{i}=\frac{\partial \Pi}{\partial \phi_{l}^{i}}=\sum_{W_{l}} \int_{\Omega_{W_{l}}} \frac{\partial N_{l}^{i}}{\partial x^{a}}\left(u_{l}^{a}-u_{u}^{a}\right) d \Omega_{W_{l}}
\end{aligned}
$$

and their corresponding Jacobians: 


$$
\begin{aligned}
& J_{u u}^{i j}=\frac{\partial R_{u}^{i}}{\partial \phi_{u}^{j}}=\sum_{W_{u}} \int_{\Omega_{W_{u}}} \frac{\partial N_{u}^{i}}{\partial x^{a}} \frac{\partial N_{u}^{j}}{\partial x^{a}} d \Omega_{W_{u}}, J_{u l}^{i j}=\frac{\partial R_{u}^{i}}{\partial \phi_{l}^{j}}=-\sum_{W_{u}} \int_{\Omega_{W_{u}}} \frac{\partial N_{u}^{i}}{\partial x^{a}} \frac{\partial N_{l}^{j}}{\partial x^{a}} d \Omega_{W_{u}} \\
& J_{l u}^{i j}=\frac{\partial R_{l}^{i}}{\partial \phi_{u}^{j}}=-\sum_{W_{l}} \int_{\Omega_{W_{l}}} \frac{\partial N_{l}^{i}}{\partial x^{a}} \frac{\partial N_{u}^{j}}{\partial x^{a}} d \Omega_{W_{l}}, J_{l l}^{i j}=\frac{\partial R_{l}^{i}}{\partial \phi_{l}^{j}}=\sum_{W_{l}} \int_{\Omega_{W_{l}}} \frac{\partial N_{l}^{i}}{\partial x^{a}}, \frac{\partial N_{l}^{j}}{\partial x^{a}} d \Omega_{W_{l}}
\end{aligned}
$$

\section{RESULTS}

To evaluate the proposed embedded wake approach, the flow over a two-dimensional NACA0012 airfoil and a three-dimensional rectangular wing are computed. Lift, pitching moment, and pressure coefficients are compared with reference data from other codes.

Salome is used to generate the geometries and the meshes [5]. A significant advantage of using an embedded wake approach is that the wake does not need to be explicitly modeled in the domain, substantially reducing the modeling time. In the numerical studies presented here, triangular and tetrahedral meshes are created using the NETGEN algorithm [6]. The main parameters defining the meshes are the maximum element size, the minimum element size, and the growth rate, which is the maximum ratio by which two adjacent elements can differ of two adjacent elene
into Kratos' input ne
employed [7]. Using th
has been developed.

\subsection{NACA 0012}
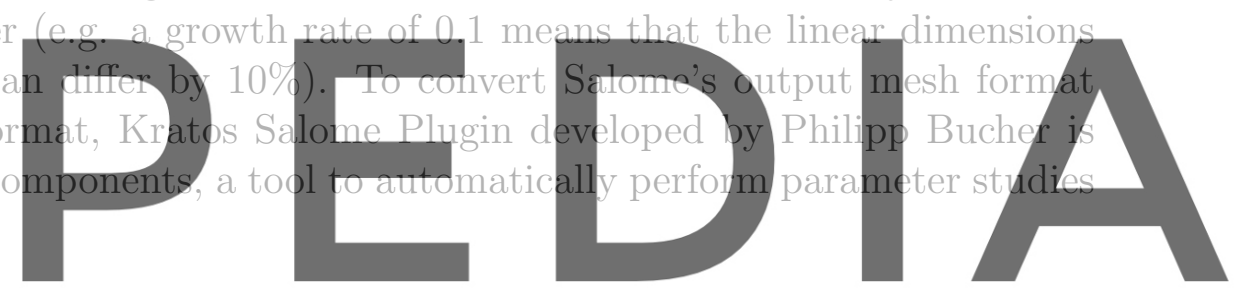

Register for free at https//www.scipedia.com to download the version without the watermark

ficients is investigated. The airfoil's shape is described by:

$$
z= \pm 0.6\left(0.2969 \sqrt{x}-0.1260 x-0.3516 x^{2}+0.2843 x^{3}-0.1015 x^{4}\right)
$$

where $z$ is the half-thickness, and $x$ the position along the chord $(x \in[0,1])$. This description results in a blunt trailing edge. For the purposes of this study, the last coefficient was slightly modified to achieve a sharp trailing edge:

$$
z= \pm 0.6\left(0.2969 \sqrt{x}-0.1260 x-0.3516 x^{2}+0.2843 x^{3}-0.1036 x^{4}\right)
$$

This modification is specified by the AIAA Aerodynamic Design Optimization Discussion Group. The airfoil was generated using Salome's parametric curve generator, which defines a parametric equation for each coordinate. In total, six meshes with a domain size of $100 \mathrm{~m}$ and a chord length of $1 \mathrm{~m}$ are considered. Figure 3 shows the domain's mesh in the far-field and in the vicinity of the airfoil for different mesh refinements, where the minimum element size is denoted by h. Note the substantial benefit of using an embedded 
I. López, E. Baez Jones, M. Nuñez, R. Zorrilla, R. Rossi, K.-U. Bletzinger and R. Wüchner

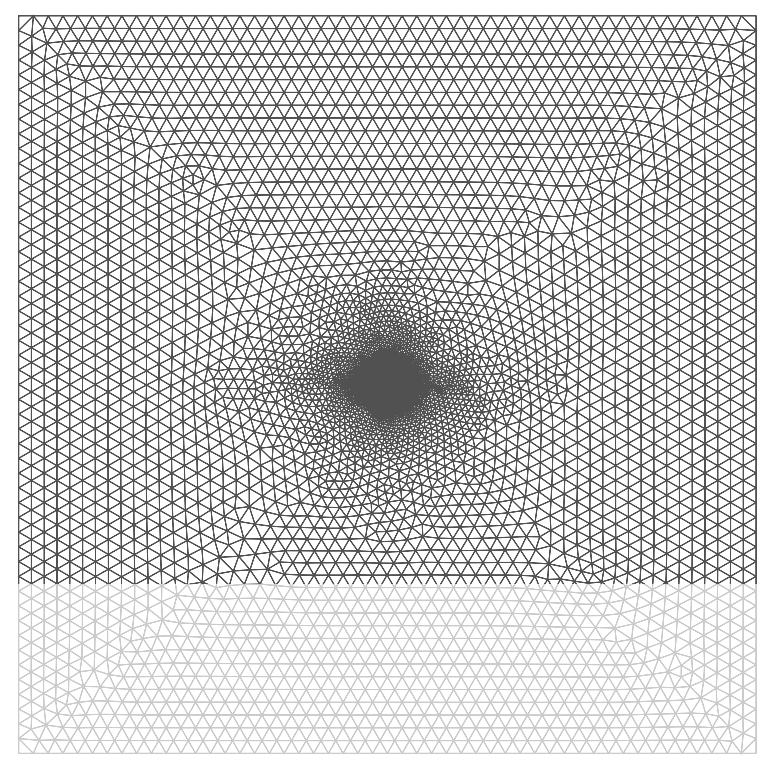

(a) Domain's far-field mesh.
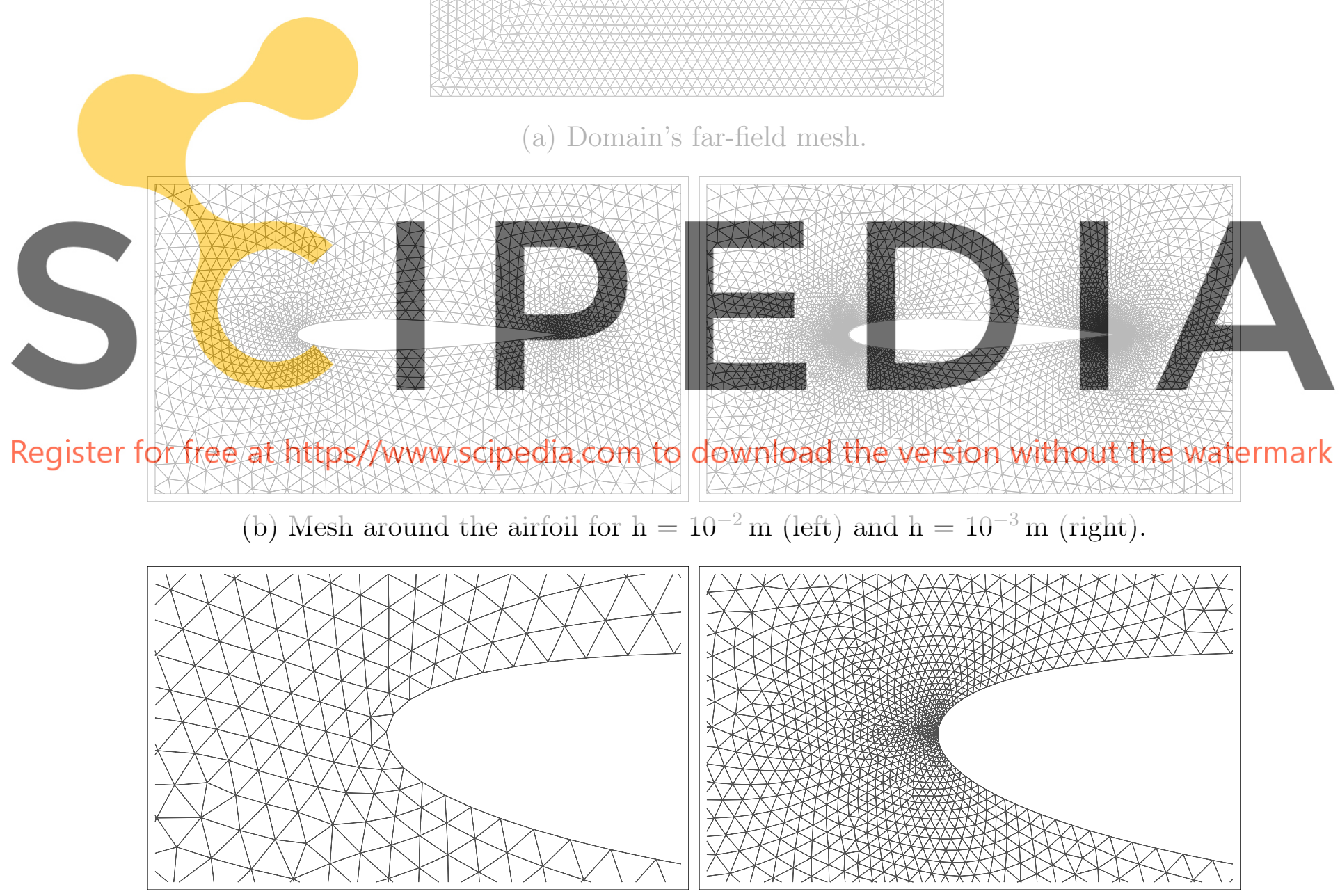

(c) Mesh around the leading edge for $\mathrm{h}=10^{-2} \mathrm{~m}$ (left) and $\mathrm{h}=10^{-3} \mathrm{~m}$ (right).

Figure 3: Meshes around NACA 0012. 
wake approach, where the wake does not need to be explicitly represented by the mesh. The meshes are refined towards the leading and trailing edge, where the solution's gradients are largest. This refinement also accurately captures the geometry at the leading edge, where the radius of curvature is smallest. The airfoil is discretized using a geometric progression where the common ratio is set to 1.01. The domain's boundary is discretized using a uniform distribution with an element size of $2 \mathrm{~m}\left(1 / 50^{\text {th }}\right.$ of the domain size). The domain's maximum element size matches the boundary's element size, and the mesh growth rate is set to 0.05 to ensure a smooth element size transition.

Figures 4 and 5 present the convergence of the lift and pitching moment coefficients, and their errors over the minimum element size. The lift coefficient's relative error is expressed as a percentage using:

$$
\delta_{c_{l}}=\frac{\left|c_{l}-c_{\text {lref }}\right|}{\left|c_{\text {lref }}\right|} \cdot 100 \%
$$

where the reference lift coefficient $\left(c_{\text {lref }}=0.2038\right)$ is the value reported in [8]. The moment coefficient is computed using a near-field analysis, whereas the lift coefficient is computed using three approaches:

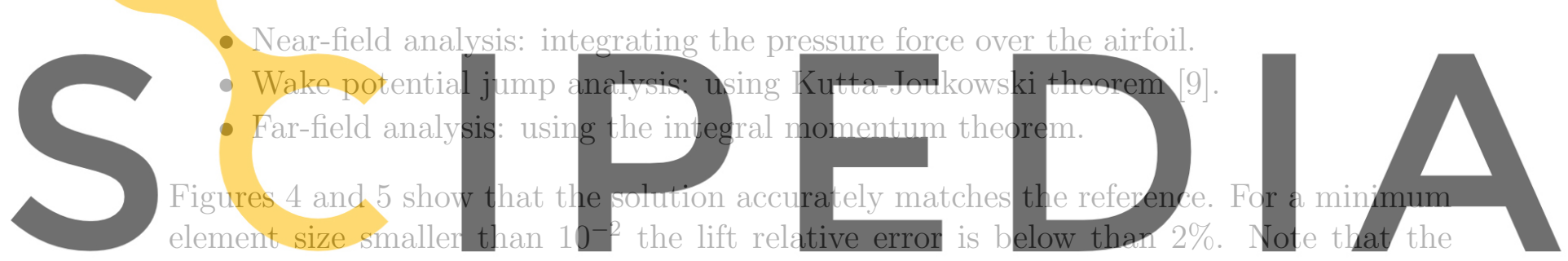

three force analysis yield very similar results.

Register for free at https//www.scipedia.com to download the version without the watermark Lift coefficient
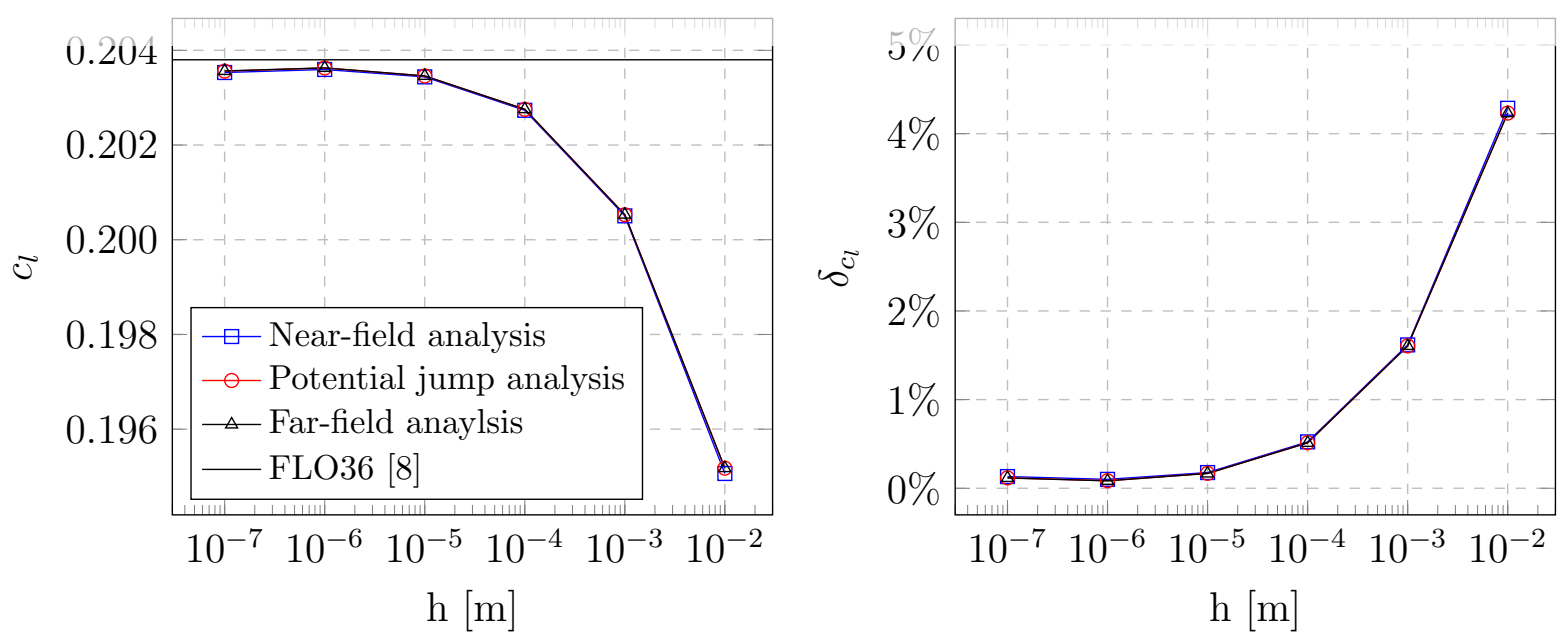

Figure 4: NACA 0012 mesh refinement study $\left(\alpha=1^{\circ}, M_{\infty}=0.72\right)$. 
I. López, E. Baez Jones, M. Nuñez, R. Zorrilla, R. Rossi, K.-U. Bletzinger and R. Wüchner
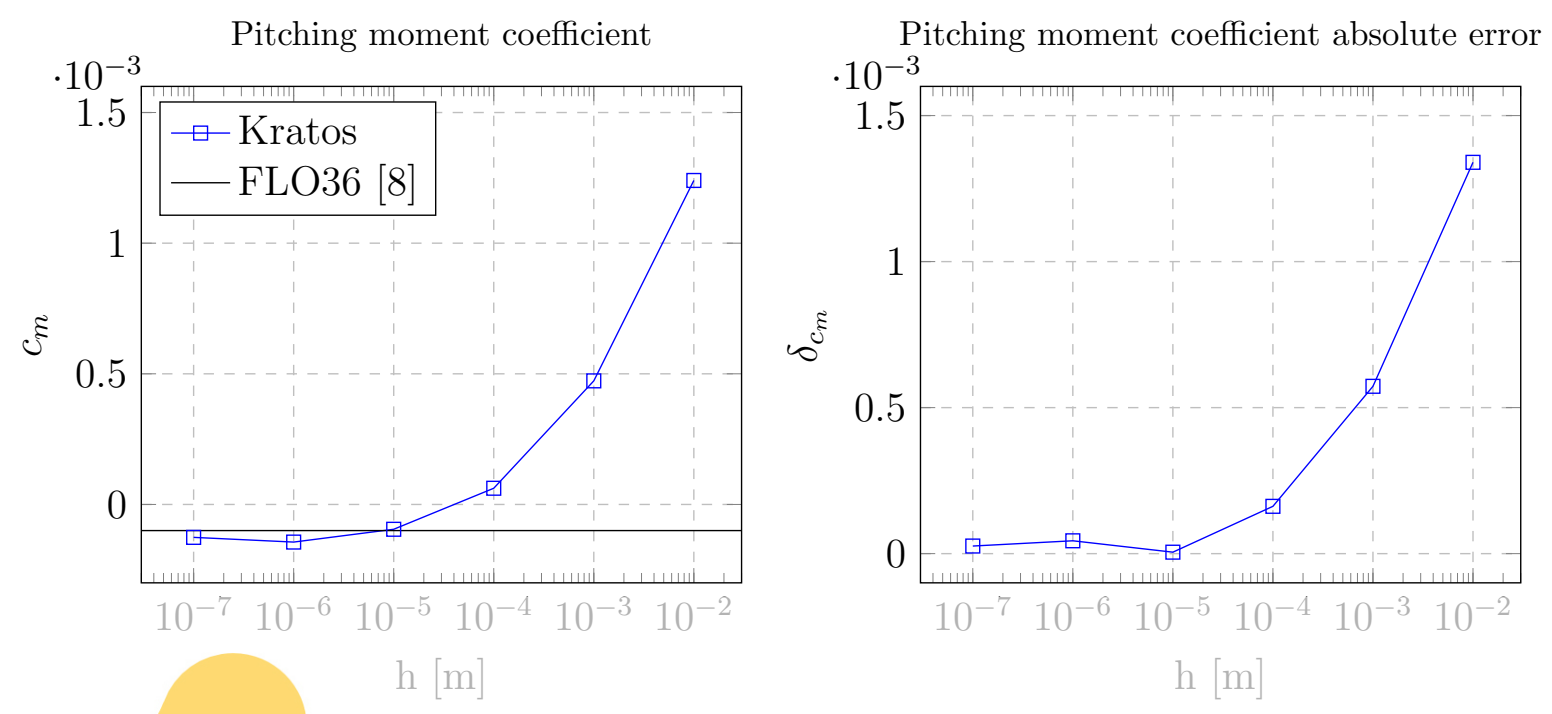

Figure 5: NACA 0012 mesh refinement study $\left(\alpha=1^{\circ}, M_{\infty}=0.72\right)$.
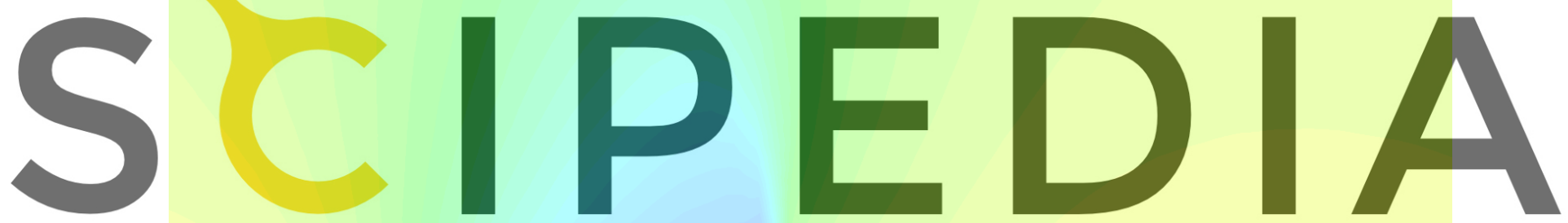

Register for free at https//www.scipedia.com to download the version without the watermark

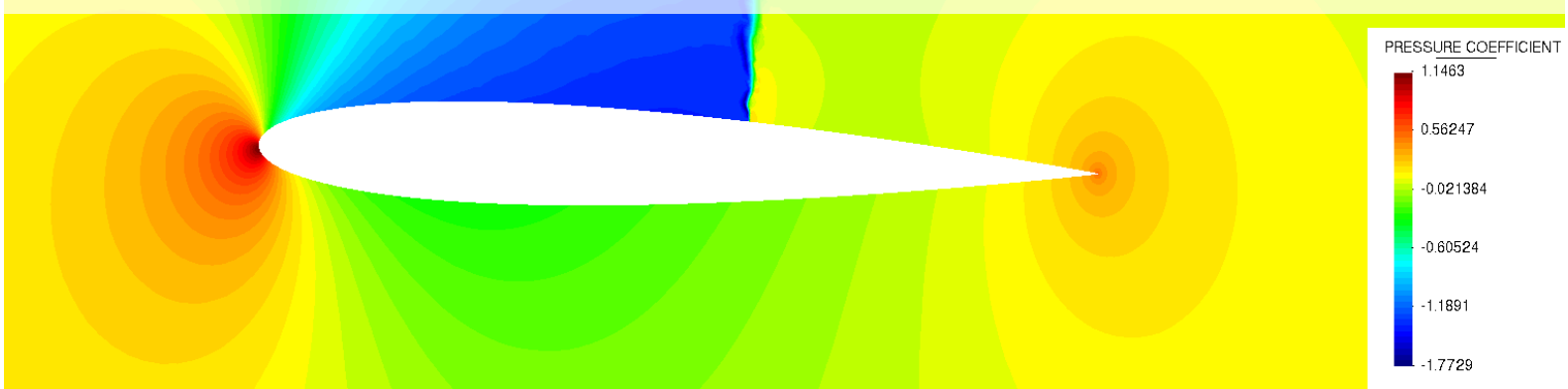

Figure 6: Pressure coefficient contour plot, NACA $0012\left(\alpha=2.0^{\circ}, M_{\infty}=0.75\right)$.

Figure 6 shows the pressure coefficient contour plot for $\alpha=2.0^{\circ}$ and $M_{\infty}=0.75$. The pressure coefficient is continuous across the wake, meaning that the wake pressure equality boundary condition is satisfied. Figure 7 compares the solution and reference pressure 


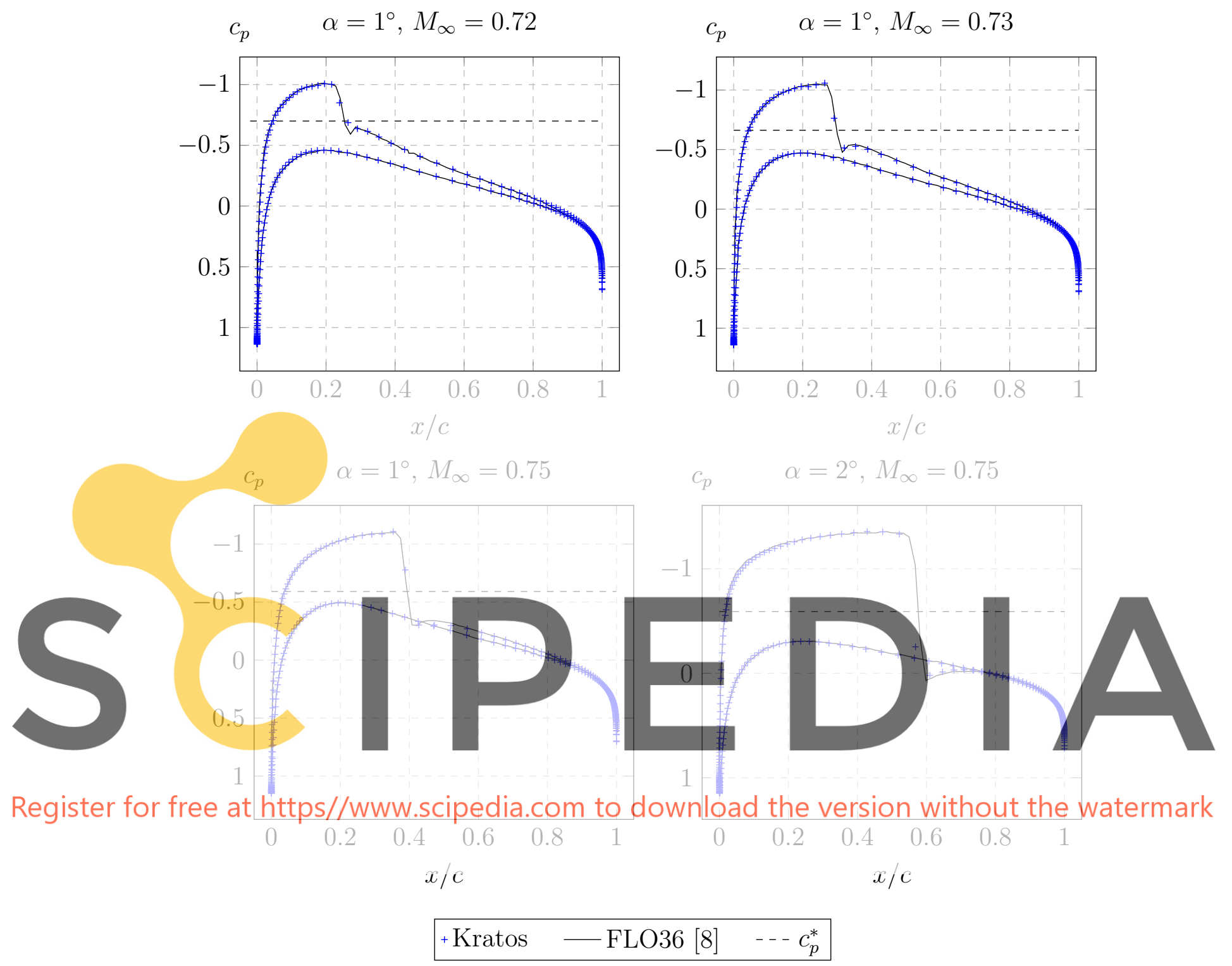

Figure 7: Pressure coefficient distributions.

coefficient distributions along the upper and lower airfoil surfaces for the different flow conditions. Note that despite using an embedded wake approach, the stagnation point at the trailing edge is captured accurately.

\subsection{Rectangular wing with NACA 0012 airfoil section}

The proposed method has been extended to three dimensions. To verify the implementation, the solution of a rectangular wing of aspect ratio 4 in incompressible flow at $\alpha=5^{\circ}$ is presented. Figure 8a shows the wing's surface mesh and a clip of the tetrahedral 


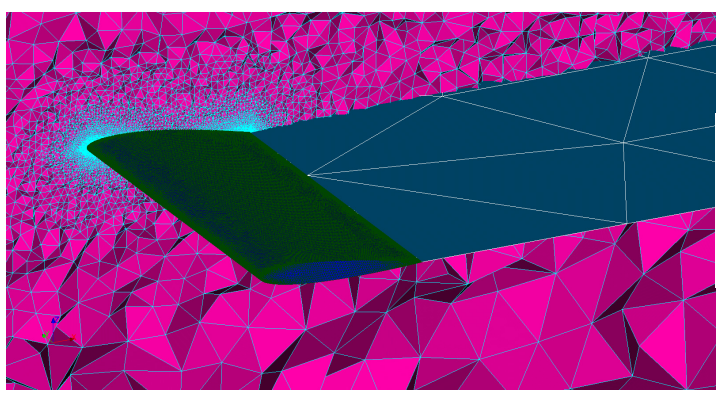

(a) Wing's mesh.

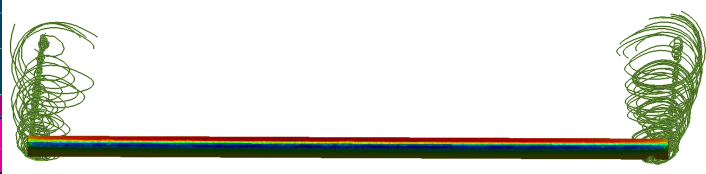

(b) Tip vortices' structure.

Figure 8: NACA0012 rectangular wing.
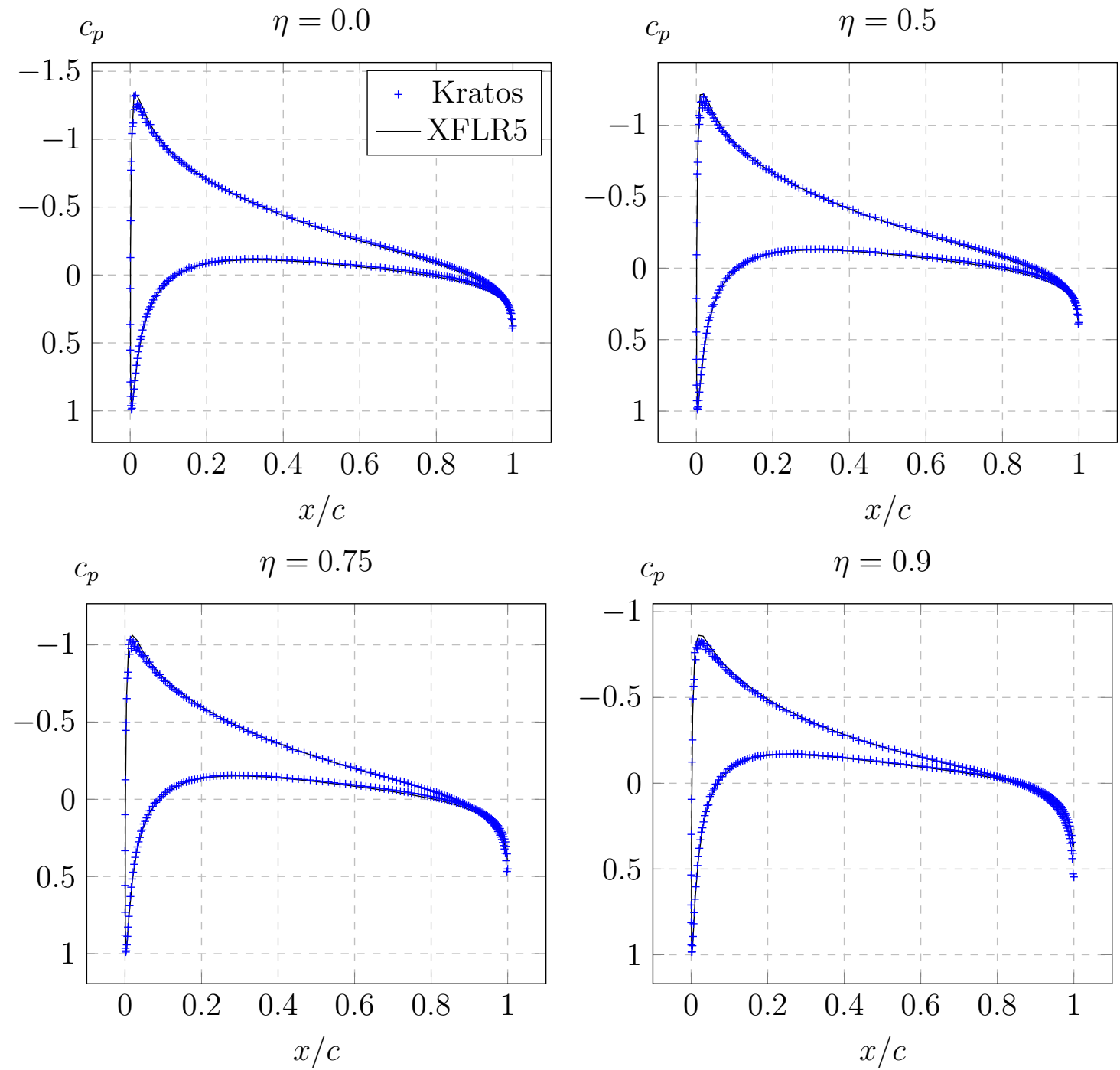

Figure 9: Pressure coefficient distribution at different wing sections. 
volume mesh along the symmetry plane. The wake surface is indicated in blue. Figure 8b shows a front view of the wing along with the tip vortices' structure. In Figure 9 the pressure coefficient distribution is compared with the solution from XFLR5 at four wing sections, where each section spanwise location $y$ is nondimentionalized using the half span $b(\eta=y / b)$.

\section{CONCLUSIONS}

An embedded wake approach for a potential transonic solver is presented. This approach allows reducing the modeling time significantly. Furthermore, the method's robustness is demonstrated for two- and three-dimensional examples, where the reference data is accurately reproduced without requiring an extra mesh refinement towards the wake. Describing the wake implicitly within the mesh allows to effectively perform aeroelastic optimization, where the wake's position may change due to the structural response and the geometry's evolutionary steps. In the future, this approach will be verified for three-dimensional transonic flow conditions.

\section{REFERENCES}

[1] David Eller. "Fast, Unstructured-Mesh Finite-Element Method for Nonlinear Subsonic Flow". In: Journal of Aircraft 49.5 (2012), pp. 1471-1479. ISSN: 0021-8669. DOI: 10.2514/1.c031738.

[2] B. Nishida. "Fully simultaneous coupling of the full potential equation and the integral boundary layer equations in three dimensions". In: 1996.

[3] Mark Drela. Flight Vehicle Aerodynamics. 2013. IsBn: 9780262526449.

[4] M. Hafez, J. South, and E. Murman. "Artificial Compressibility Methods for Numerical Solutions of Transonic Full Potential Equation". In: AIAA Journal 17.8 (1979), pp. 838-844. DOI: 10.2514/3.61235.

[5] C. Caremoli and A. Ribes. "Salome platform component model for numerical simulation". In: 2013 IEEE 37th Annual Computer Software and Applications Conference. Vol. 2. Los Alamitos, CA, USA: IEEE Computer Society, 2007, pp. 553-564. DOI: 10.1109/COMPSAC. 2007.185.

[6] Joachim Schoeberl. "NETGEN An advancing front 2D/3D-mesh generator based on abstract rules". In: Computing and Visualization in Science 1 (July 1997), pp. 41-52. DOI: $10.1007 / \mathrm{s} 007910050004$.

[7] Philipp Bucher. KratosSalomePlugin. https://github.com/KratosMultiphysics/ KratosSalomePlugin. 2020.

[8] G. Volpe and A. Jameson. "Transonic potential flow calculations by two artificial density methods". In: AIA A Journal 26.4 (1988), pp. 425-429. DoI: 10.2514/3.9910.

[9] Joseph Katz and Allen Plotkin. Low-Speed Aerodynamics. Cambridge University Press, 2001. ISBN: 9780521665520. DOI: 10.1017/CB09780511810329. 